\title{
BASIC EXAMINATION OF FUTURE CHANGE OF TROPICAL CYCLONE AND STORM SURGE PROPERTIES UNDER CLIMATE CHANGE
}

\author{
Sota Nakajo ${ }^{1}$, Nobuhito Mori ${ }^{2}$, Tomohiro Yasuda ${ }^{2}$, Hajime Mase $^{2}$
}

\begin{abstract}
Recently high-resolution Global Climate Model (GCM) results show that future Tropical Cyclone (TC) characteristics, such as arrival frequency, developing process and intensity, will be changed by global climate changes. However, there are major two difficulties for assessment of future TC disaster. One is uncertainty of future projection of GCM, and another is shortage of sample TC data for statistical analysis. In this paper, we generated many synthetic TC data with Global Stochastic Tropical Cyclone Model (GSTCM) and estimated future changes of TC properties and reduced uncertainty by ensemble averaging of multi-GCM projection results. In addition, GSTCM which have empirical temporal correlation algorithm was improved for the reproducibility of arrival TC statistics by cluster analysis of TC data. This upgrade could pave the way to local future projection of TC disaster.
\end{abstract}

Keywords: Tropical cyclone; Monte-Calro simulation; Cluster analysis; Climate change

\section{INTRODUCTION}

Recently high-resolution Global Climate Model (GCM) results show that future Tropical Cyclone (TC) characteristics, such as arrival frequency, developing process and intensity, will be changed by global climate changes. For example, Knutson et al. (2010) concluded the global cyclogenesis frequency will be decreased or remained essentially unchanged, and the mean maximum wind speed of TC will be increased globally and maybe the frequency of the most intense TC will increase. External forces caused by TC in long return period are important for designs and maintenances of coastal structures. Therefore, next we need more local information of future change of TC. However, we have two difficulties for assessment of future TC disaster. One is uncertainty of future projection of GCM. This uncertainty was caused by natural trend and anthropogenic factors such as greenhouse gas effect. And another is shortage of sample TC data. Actually, the extreme severe TC disaster frequency is very low; moreover disaster damage is sensitive to TC track. This problem is same even in numerical simulation of TC. GCM result with enough spatial resolution is not realistic to be applied to practical design directly. For example, the number of TC which is approaching to Tokyo bay during 25 years was only three in simulation result of MRI-AGCM (Meteorological Research Institute, Japan).

One of approach to increase the number of TC event for particular region is use of a stochastic downscaling method which can generate numerous artificial TC data which cannot obtain by physical model. This stochastic method implemented Monte-Carlo simulation is called Stochastic Tropical Cyclone Model (STCM). In the STCM, sequential development of TC is calculated statistically from given statistical property of TC data. Several STCMs were proposed in the past. Most major method is an autoregression model (AR model) which expresses TC properties in sum of polynomial equation and random term (Vickery et al. (2000), James and Mason (2005), Hall and Jewson (2007)). Generally, AR model low reproducibility of seasonal change or extreme TC, although average TC property gives good agreement with data. To expand AR model limitation, classification of TC data was attempted in many studies and AR model coefficients are decided at every class to improve model accuracy. However these classifications were performed in an arbitrary manner, therefore generalization of AR model is basically difficult. In addition, in previous studies, STCM was developed for specific ocean basin and global model have never been developed. One major reason may be those AR models have arbitrariness in classification of TC data.

In this study, we propose new temporal correlation Probability Density Function (PDF) type Global STCM (GSTCM) adapting using available all TC data and verify its validation is carried out. Moreover, we estimate future changes of TC properties and reduced the uncertainty of future projection by ensemble averaging of multi-GCM results, and implement them to GSTCM. Finally, future change of average and extreme TC and storm surge characteristics are shown in this paper.

\section{METHODOLOGY}

\section{Overview of Global Stochastic Tropical Cyclone Model}

In our model, GSTCM, TC track and development and decay process of TC are recreated globally. Fig. 1 shows flow chart of GSTCM. In this study, International Best Track Archive for Climate Steward-

\footnotetext{
${ }^{1}$ Department of Civil and Environmental Engineering, Kumamoto University, Japan

${ }^{2}$ Disaster Prevention Research Institute, Kyoto University, Japan
} 


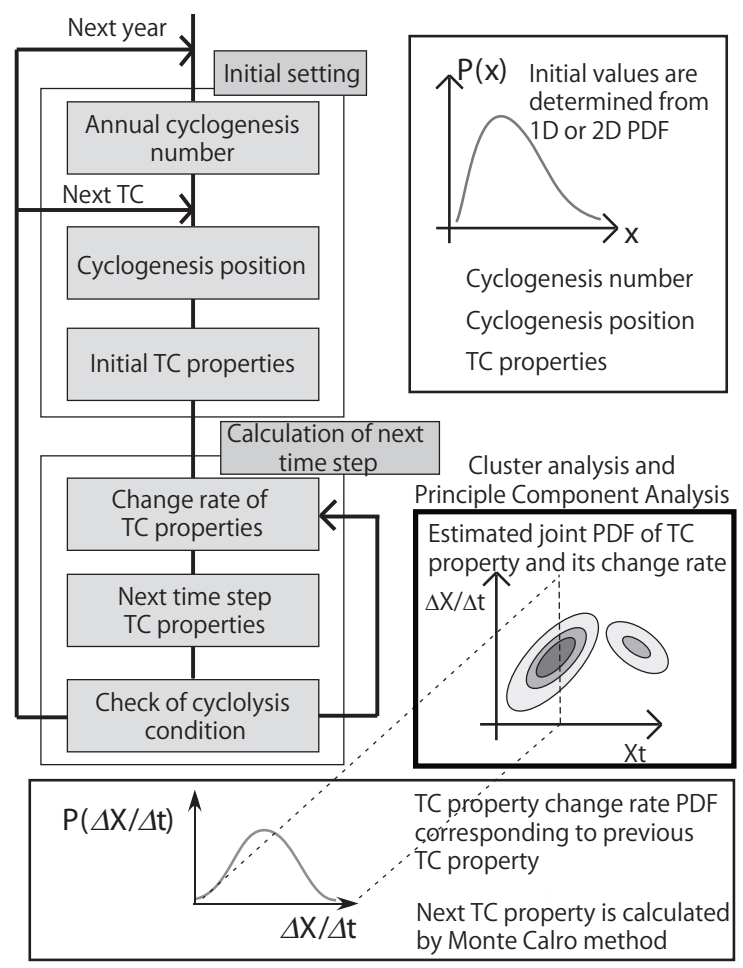

Figure 1: Flow chart of Global Stochastic Tropical Cyclone Model (GSTCM)

ship.v02r01 (IBTrACS) proposed by National Oceanic and Atmospheric Administration (NOAA) was used as source data as a calibration of statistical model coefficients. IBTrACS.v02r01 is global unified observation dataset from 1800s until 2008. This data confirmed ensemble mean data passed reliability check when multi-institute and multi-method observation data were unified as the same event. IBTrACS includes longitude and latitude of TC center, central pressure and maximum wind speed in every 6 hours. In this study, we modeled statistically three TC properties, translation direction and speed, central pressure. On the basis of IBTrACS reliability index, we excluded some part of central pressure data for modeling because they showed low reliability. The partial lack of data was filled by linear interpolation.

In our model, all given statistics were calculated at every 3 degree grid in advance and the Monte-Carlo simulation was conducted at every 1 degree grid where interpolated various statistics. GSTCM contains three parts. First part is a cyclogenesis process model. Annual global cyclogenesis number, cyclogenesis position and initial TC properties are determined statistically based on their historical data PDF with Monte-Carlo simulation. PDF of annual global cyclogenesis number can be approximated by lognormal distribution. Cyclogenesis position is determined statistically based on global cyclogenesis frequency distribution estimated from historical data at every 1 degree grid by using Monte-Carlo method. Initial TC properties are determined statistically based on each property's PDF estimated from historical data. At that time, it is assumed and validated that translation direction follows the normal distribution and translation speed and central pressure follow the lognormal distribution respectively.

Second part is a developing process model. This model provides TC properties change rate as function of previous property value. Therefore, subsequent TC properties are calculated from joint PDF of TC property and its change rate at each location. The joint PDF is approximated based on Principle Component Analysis (PCA) of historical data. That is, two normal distributions along two principle components axis were synthesized. Therefore, arbitrary change rate PDF is calculated by each previous time step TC property value from this joint PDF. Then the change rate of TC properties are determined statistically with MonteCarlo simulation, and next time step TC properties are calculated from multiplication of the change rate and time increment.

Final part is a cyclogenesis process model. The cyclolysis condition is checked and if TC is survived, 
calculation goes to next time step. The criterion of cyclolysis assume following three conditions.

1. TC moves into area where no TC arrived in the past.

2. Central pressure of TC reaches to $1015 \mathrm{hPa}$ which is same to environmental atmospheric pressure

3. Cyclolysis condition is determined by statistical judgment based on cyclolysis probability $R_{\text {lysis }}$ estimated from observational data

In the case that one of these three conditions is satisfied, the corresponding TC regarded as diminished. Cyclolysis probability $R_{l y s i s}$ is determined as the ratio of cyclolysis number $N_{\text {lysis }}$ to all arrival TC number $N_{\text {arrival }}$ in each area. However in actual simulation, $R_{l y s i s}$ is determined using following equation empirically because historical data number is not enough and excess $R_{\text {lysis }}$ is estimated.

$$
\begin{gathered}
R_{\text {lysis }}^{*}=\frac{N_{\text {lysis }}}{N_{\text {arrival }}} \\
R_{\text {lysis }}=0.4 R_{\text {lysis }}^{*}
\end{gathered}
$$

\section{Improvement of developing process model}

In this study, we checked to applicability of bimodal joint PDF in second part of GSTCM for accuracy improvement, and cluster analysis was used to divide historical data into two groups. As mentioned above, PDF of TC property change rate is determined statistically as a function of previous time step value using Joint PDF in GSTCM. Therefore, it is important how joint PDF of each property is approximated accurately for improving the reproducibility of the model. And so, we proposed the method which can make smooth approximation of Joint PDF by using a PCA of TC data in local region in previous study (Nakajo et al. (2011)). First appropriate virtual variables are estimated from PCA in order to express the temporal correlation between TC property $\phi$ and its change rate $\dot{\phi}$. In this study, not only first principal component $\alpha$ but also secondary principal component $\beta$ is calculated in order to propose the simple model considering its correlation variance change. The values of $\alpha$ and $\beta$ are expressed by the following equations,

$$
\begin{aligned}
\alpha & =a_{1}(\phi-\bar{\phi})+a_{2}(\dot{\phi}-\overline{\dot{\phi}}) \\
\beta & =b_{1}(\phi-\bar{\phi})+b_{2}(\dot{\phi}-\overline{\dot{\phi}})
\end{aligned}
$$

here $\bar{\phi}$ and $\overline{\dot{\phi}}$ are mean values of TC property and its change rate, $\vec{a}\left(a_{1}, a_{2}\right)$ and $\vec{b}\left(b_{1}, b_{2}\right)$ are eigenvectors calculated from variance-covariance matrix of $\phi$ and $\dot{\phi}$, respectively. When we assume normal distribution of principal components $\alpha$ and $\beta$, Joint PDF are approximated as a superposition of two normal distributions around two principal axes. Therefore Joint PDF is determined the function $f\left(\bar{\phi}, \bar{\phi}, \bar{\alpha}, \bar{\beta}, \sigma_{\alpha}, \sigma_{\beta}\right)$ expressed by six explanatory variables. Here $\bar{\alpha}, \bar{\beta}, \sigma_{\alpha}, \sigma_{\beta}$ means average and standard deviation of $\alpha$ and $\beta$, respectively. An example of Joint PDF for central pressure is presented as contour lines in Fig. 2(a). This figure is one of sample data obtained at 3 degree grid centered at 8 degrees north latitude and 137 degrees east longitude. Marker plots and contour line means historical data and approximation of their Joint PDF, respectively. There is a temporal correlation between central pressure and its change rate locally . If previous central pressure is small (this means TC is strong), change rate will be likely to small (TC will be strong at next time step). The logarithmic value $\phi$ of central pressure and translation speed are used for statistical modeling. The ridge lines of this Joint PDF correspond to the principal component axes. Parameterized Joint PDF can be considered to represent appropriate distribution validated many observation data.

However, if we consider in more detail of distribution, distribution of historical data in space $p$ - $\Delta p$ can be divided to two parts where $\Delta p$ is change rate of central pressure. That is, possibly the bimodal joint PDF is better than the unimodal joint PDF which is assumed in previous model. With this feature in mind, in this study, we conducted cluster analysis of TC source data and classified two clusters first. The Joint PDF is made from each cluster and then the bimodal Joint PDF is synthesized with a weight of each cluster number ratio. We adopted K-mean method for cluster analysis algorithm, and Euclidean distance for criterion of clustering. Initial center of each cluster was selected 50 times at random, and we adopted the result when summation of distance from each center becomes minimum value. Fig. 2(b) shows the bimodal joint PDF result. As can be seen from this figure, bimodal Joint PDF has one cluster peak around 1000hPa and another 


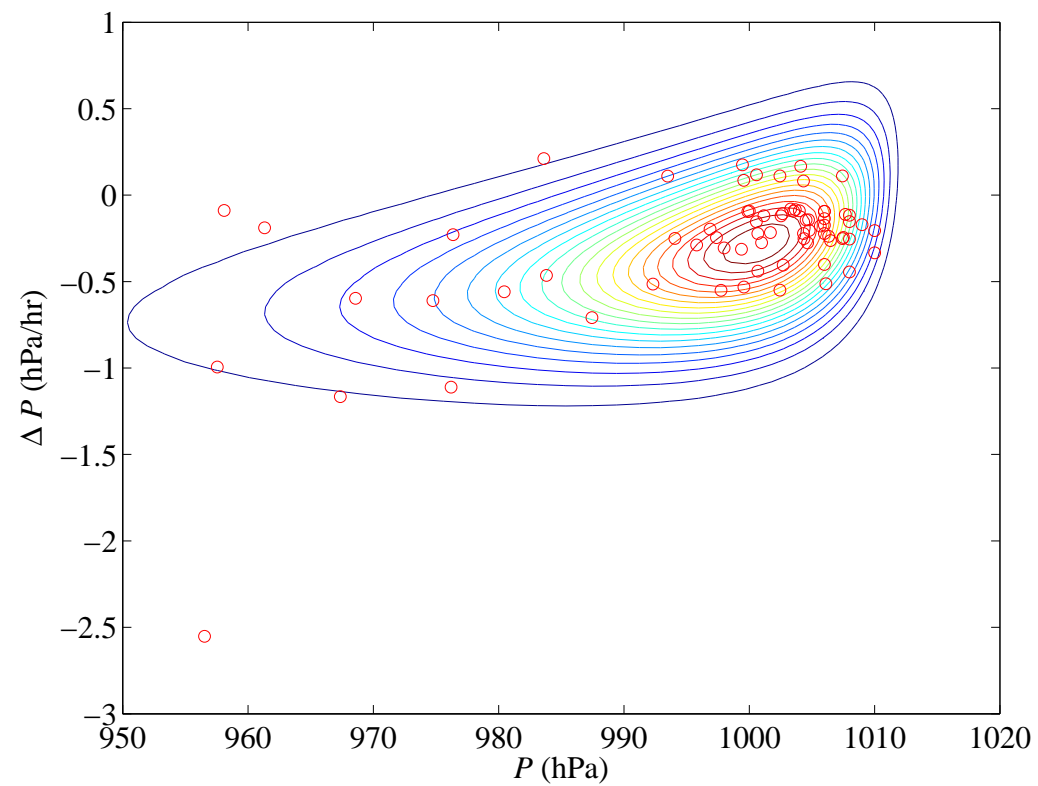

(a) Unimodal joint PDF

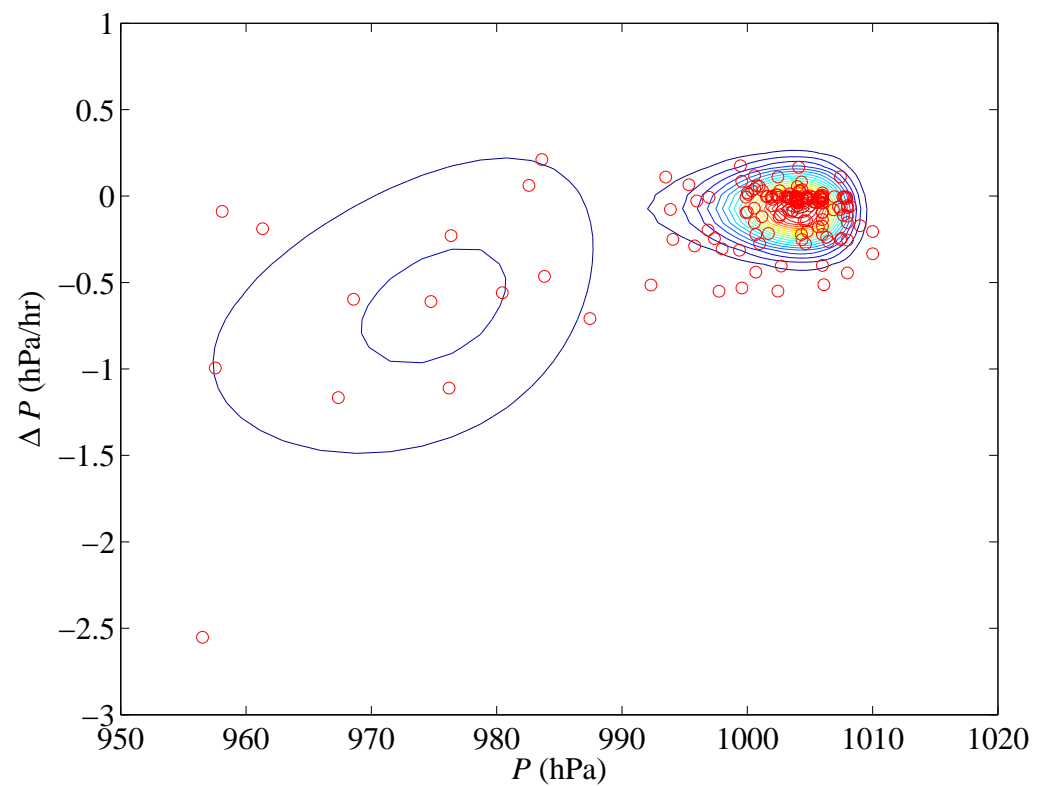

(b) Bimodal joint PDF

Figure 2: Approximation of joint PDF of TC central pressure and its change rate at $137 \mathrm{E}^{\circ}, \mathbf{8 N}^{\circ}$ 


\begin{tabular}{|c|c|c|}
\hline \multicolumn{3}{|c|}{ Table 1. Area classification for arrival TC analysis } \\
\hline Area number & East longitude & North latitude \\
\hline Area 1 & from 120 to $160 \mathrm{deg}$. & from 10 to $20 \mathrm{deg}$. \\
\hline Area 2 & from 120 to $160 \mathrm{deg}$. & from 20 to $30 \mathrm{deg}$. \\
\hline Area 3 & from 120 to $160 \mathrm{deg}$. & from 30 to $40 \mathrm{deg}$. \\
\hline Area 4 & from 120 to $160 \mathrm{deg}$. & from 40 to $50 \mathrm{deg}$. \\
\hline
\end{tabular}

\begin{tabular}{|c|c|c|c|}
\hline \multicolumn{4}{|c|}{ Table 2. Model comparison } \\
\hline Model name & Central pressure & Translation speed & Translation direction \\
\hline$U$ & Uni. & Uni. & Uni. \\
\hline$B_{p}$ & Bi. & Uni. & Uni. \\
\hline$B_{s}$ & Uni. & Bi. & Uni. \\
\hline$B_{d}$ & Uni. & Uni. & Bi. \\
\hline$B_{p s}$ & Bi. & Bi. & Uni. \\
\hline$B_{p d}$ & Bi. & Uni. & Bi. \\
\hline$B_{s d}$ & Uni. & Bi. & Bi. \\
\hline$B_{p s d}$ & Bi. & Bi. & Bi. \\
\hline \multicolumn{4}{|c|}{ Uni. - Unimodal joint PDF, Bi. ; Bimodal joint PDF }
\end{tabular}

cluster have a peak around $975 \mathrm{hPa}$. Comparing to unimodal model, the reproducibility to historical data of bimodal joint PDF is superior qualitatively.

For quantitative evaluation of model reproducibility, we checked the PDF of arrival TC property. In order to indicate relation between TC development process and reproducibility of each model, simulation data and historical data of IBTrACS were classified by arrival area shown in Table. 1 (Area 1 to 4). In this research, we compared eight models which implemented different joint PDF model by each TC property (Table. 2). Model name shows which property is modeled by bimodal joint PDF.

Comparisons of TC properties PDF (minimum central pressure, mean translation direction) of each model at each area are shown in Fig. 3 and Fig. 4. In these figures, histogram means historical data PDF and line plot means simulation results PDF. Historical data is 59 years data from 1950 to 2008 and simulations results are ensemble mean of 50 groups of 50 years data. In these figures, confidence intervals of standard deviation are also presented. As can be seen in Fig 3, bimodal model (blue colors : $B p, B p s$, $B p d, B p s d$ ) results have better reproducibility than that of unimodal model (green and red colors : $U$, $B s, B d, B s d$ ). Minimum central pressure PDF of observation data have a peak around 985 1000 hPa, and data over $985 \mathrm{hPa}$ occupied about $60 \%$ at Area 1 (Fig. 3(a)). The peaks of PDF of unimodal model results were biased toward $985 \mathrm{hPa}$ comparing to historical data. Moreover, unimodal model results were underestimated the ratio of low central pressure data $(895 \sim 940 \mathrm{hPa})$. On the other hand, bimodal model results were good agreement with historical data. This superiority of bimodal model is also shown in other area (Fig. 3(b)-(d)). The PDF of historical data was changed with increase of latitude and the ratio of moderate central pressure (940 970 hPa) became large. The important thing is that improvement of the approximation accuracy of joint PDF has large contribution to high reproducibility of central pressure PDF of bimodal model. Furthermore, it becomes obvious that minimum central pressure PDF depends on Joint PDF of central pressure dominantly and other modeling method for translation direction and speed are not significantly influenced to it.

The improvement of GSTCM by implementation of bimodal joint PDF is also shown in the PDF of mean translation direction (Fig. 4). Translation direction is defined so as to increase in a clockwise direction and it is 0 when they face to the north. Consequently, bimodal model results (blue : $B d, B p d, B s d, B p s d$ ) show higher reproducibility than that of unimodal model results (green or red : $U, B p, B s, B p s$ ). At Area 1 (Fig. 4(a)), most TC propagates toward 270 degree direction (westward) and PDF distribution was slightly biased to north in historical data. All model results almost reproduced this PDF distribution. The PDF distribution pattern moves to clockwise direction with increase of latitude (Fig. 4(b)-(d)). Simulation results shows similar distribution pattern but they are slightly biased to north-west direction comparing 

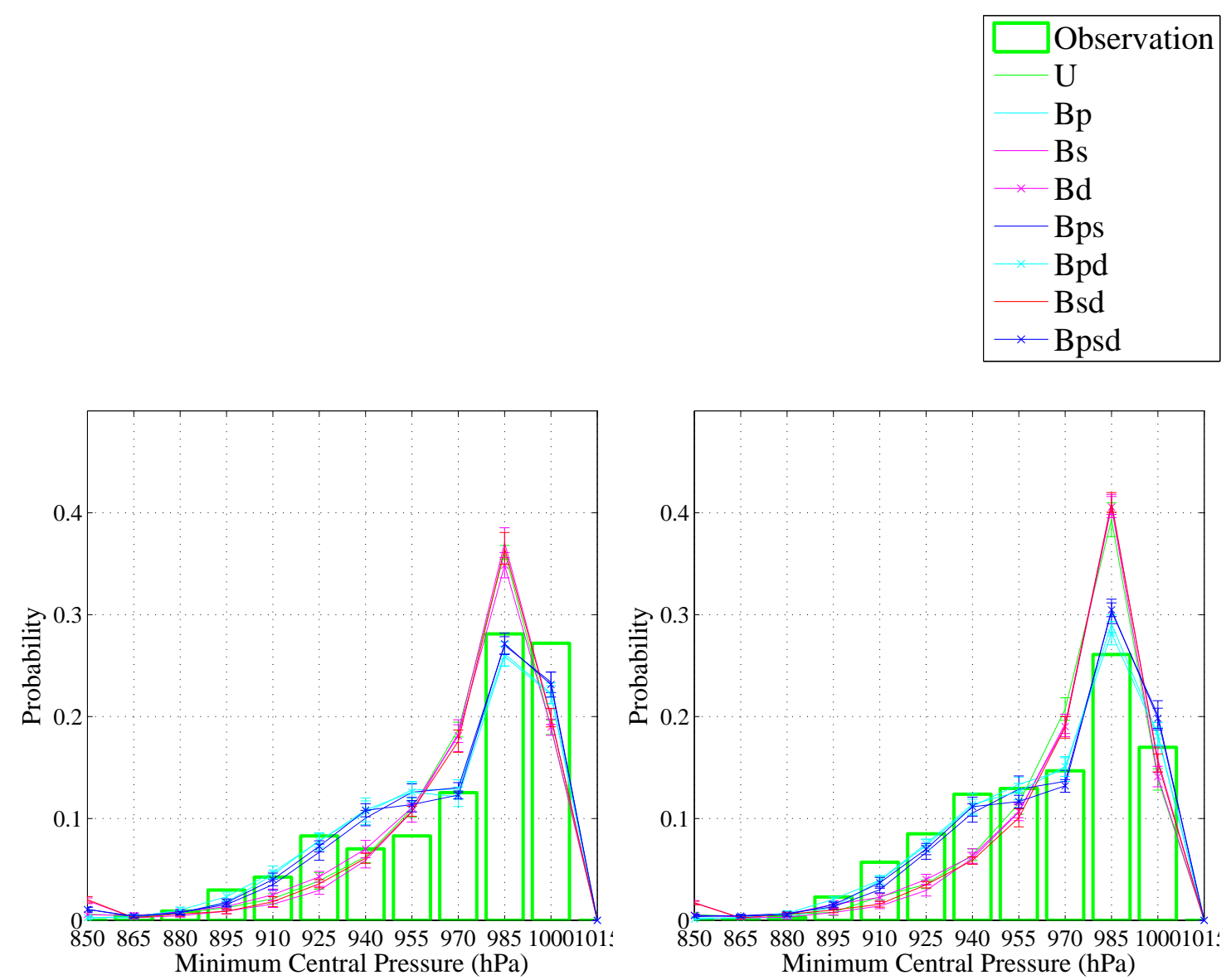

(a) Area 1

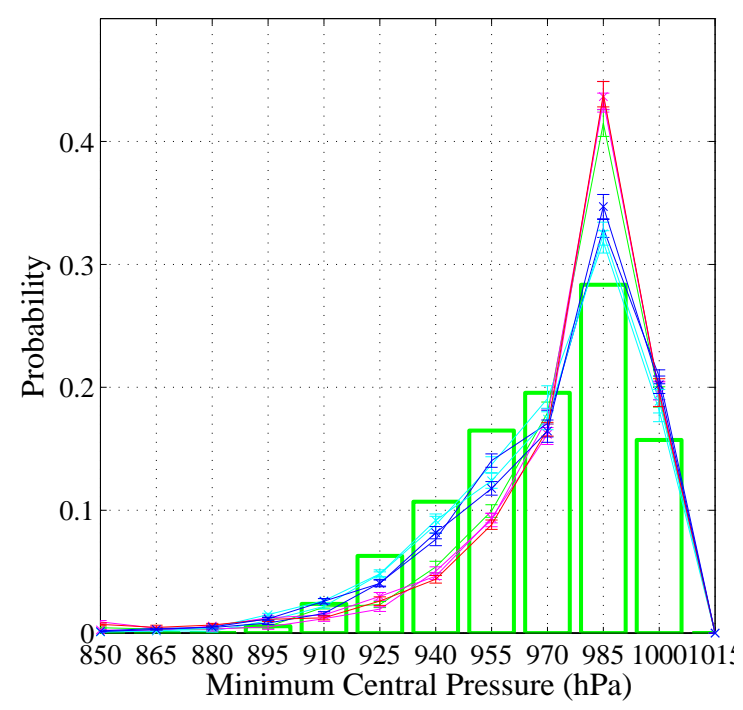

(c) Area 3

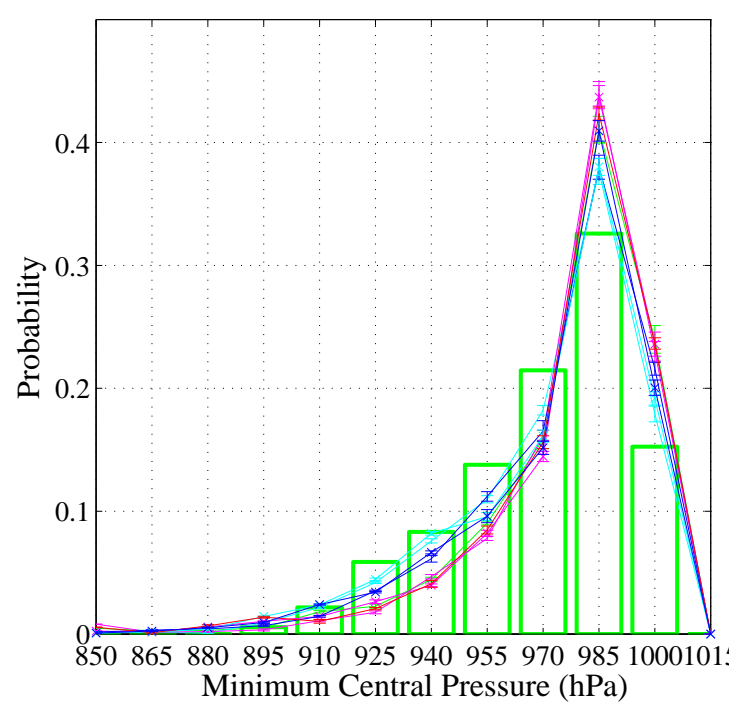

(d) Area 4

Figure 3: Arrival probability of minimum central pressure at each area 


\begin{tabular}{|c|}
\hline \multirow[t]{2}{*}{ Observation } \\
\hline \\
\hline Bp \\
\hline$x-\mathrm{Bs}$ \\
\hline $\mathrm{Bd}$ \\
\hline -Bps \\
\hline$-\quad \mathrm{Bpd}$ \\
\hline Bsd \\
\hline${ }^{*}$ Bpsd \\
\hline
\end{tabular}

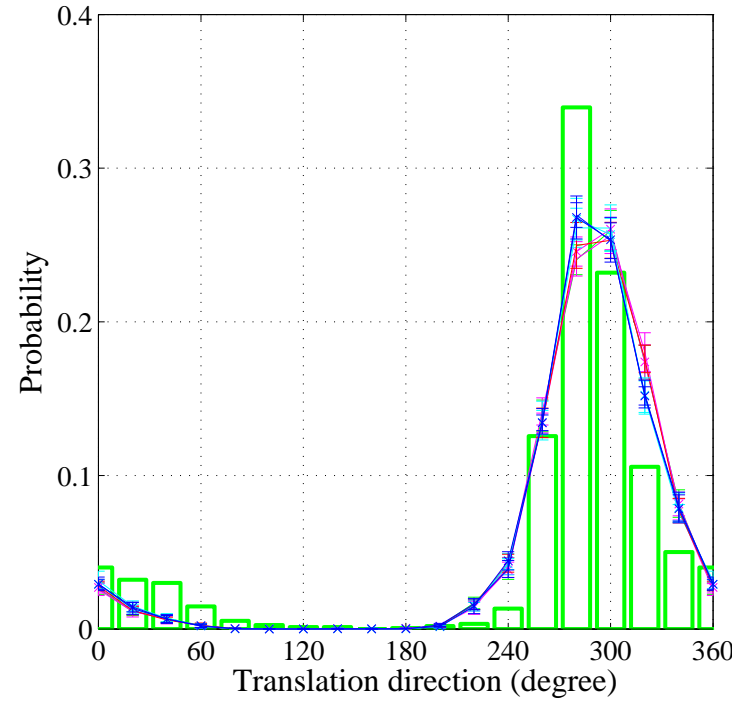

(a) Area 1

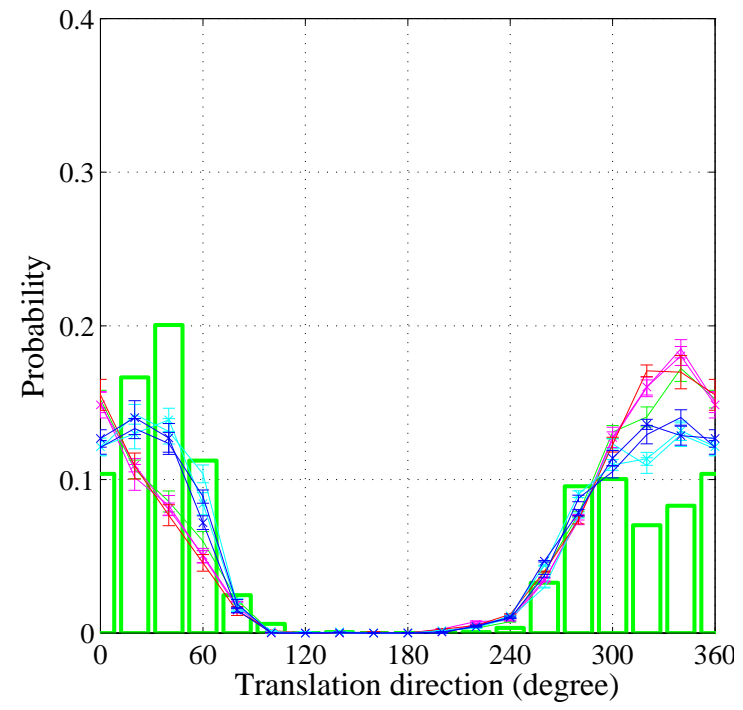

(c) Area 3

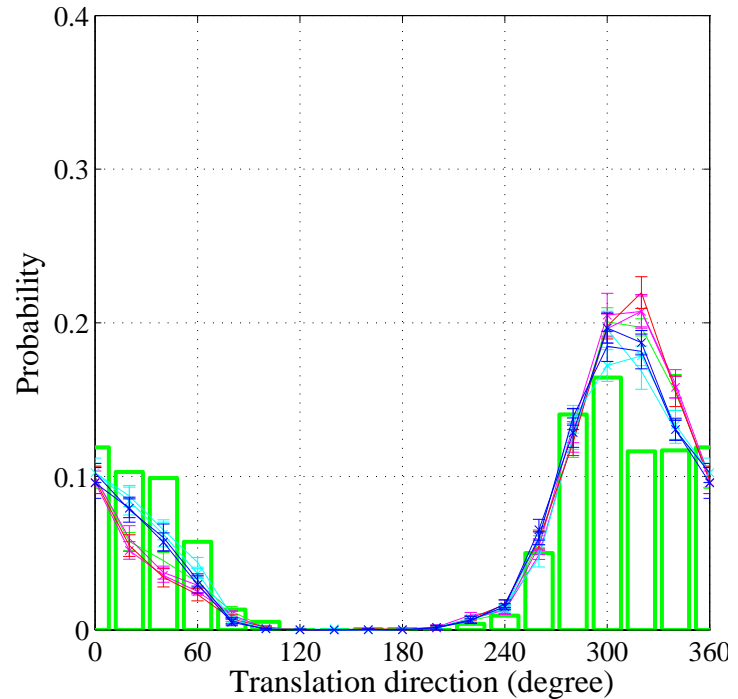

(b) Area 2

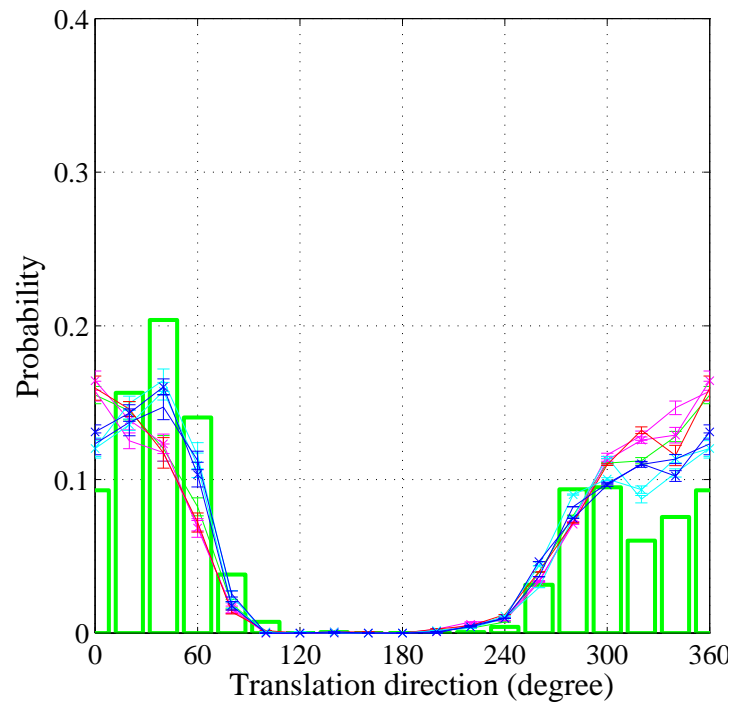

(d) Area 4

Figure 4: Arrival probability of average translation direction at each area 


\begin{tabular}{|c|c|c|c|c|}
\hline \multicolumn{5}{|c|}{ Table 3. Simulation conditions of General Circulation Model } \\
\hline Case & Period & Resolution & Cumulus convection model & Sea surface temperature \\
\hline SP0A & \multirow{6}{*}{$\begin{array}{c}\text { from } 1979 \\
\text { to } 2003\end{array}$} & $20 \mathrm{~km}$ & A-S & \multirow{6}{*}{ Observation } \\
\hline SPA & & & $\mathrm{Y}$ & \\
\hline HP0A & & \multirow{4}{*}{$60 \mathrm{~km}$} & A-S & \\
\hline HPA & & & $\mathrm{Y}$ & \\
\hline HPA kf & & & $\mathrm{K}-\mathrm{F}$ & \\
\hline HPA as & & & M. A-S & \\
\hline SF0A & \multirow{6}{*}{$\begin{array}{c}\text { from } 2075 \\
\text { to } 2099\end{array}$} & $20 \mathrm{~km}$ & A-S & \multirow{3}{*}{ M } \\
\hline SFA & & & $\mathrm{Y}$ & \\
\hline HFOA & & \multirow{4}{*}{$60 \mathrm{~km}$} & A-S & \\
\hline HFA $(a, b, c, d)$ & & & $\mathrm{Y}$ & $(\mathrm{M}, \mathrm{C} 1, \mathrm{C} 2, \mathrm{C} 3)$ \\
\hline HFA kf (a, b, c, d) & & & $\mathrm{K}-\mathrm{F}$ & $(\mathrm{M}, \mathrm{C} 1, \mathrm{C} 2, \mathrm{C} 3)$ \\
\hline HFA as $(a, b, c, d)$ & & & M. A-S & $(\mathrm{M}, \mathrm{C} 1, \mathrm{C} 2, \mathrm{C} 3)$ \\
\hline
\end{tabular}

to historical data. But the reproducibility of bimodal model results is better than that of unimodal model results.

On the other hand, the reproducibility of translation speed was not improved by implementation of bimodal joint PDF, but its detail is omitted in this paper. Therefore, we decided the model $B_{p d}$ is a most appropriate model eventually.

\section{Future change trends of TC estimated from multi-GCM results}

Essentially, future projection of TC disaster should be conducted directly by GSTCM which is calibrated based on future projection of GCM. However, future projection period of GCM is short to GSTCM calibration, and GCM result is sensitive to initial condition and physical submodel. Therefore we estimated future change trends of TC from multi-GCM results by ensemble average process and implemented them into GSTCM. We used simulation results of Atomospheric GCM, MRI-AGCM, which are developed by Meteorological Research Institute in Japan. GCM simulations were done with different resolution, cumulus convection model and sea surface temperature conditions (Table 3). In the case of HFA, HFA kf and HFA as, 4 cases of sea surface temperature were set respectively.

Future change of cyclogenesis numberTable 4 shows annual mean global cyclogenesis number simulated by GCM at present and future climate condition. Spatial resolution of GCM is not sensitive to cyclogenesis number. On the other hand, cumulus convection model affects to standard deviation of cyclogenesis number. For example, the results of Arakawa-Schubert model show relatively large yearly variation in present climate condition. Future change trend is same in all case, which is to say that the future cyclogenesis number will decrease. However, the quantities of future change of each model are different. For example, maximum future change is $-11 \%$ in case of HFA as (d) and minimum future change is $-25 \%$ in case of HFA (c). On the other hand, standard deviation of cyclogenesis number will not be changed. With these result in mind, we calculated ensemble average future change and assumed about $17 \%$ cyclogenesis number will decrease on average in this study.

Future change of TC trackFuture change of cyclogenesis location has been studied in previous studies. For example, Murakami et al. (2011) calculated future change of cyclogenesis frequency at the North Atlantic (NA) basin, and they reported the frequency of TC occurrence will decrease in the tropical western NA and increase in the tropical eastern NA. However, total development process of TC from cyclogenesis to cyclolysis was not so much having been studied. In this study, first we separated TC data to 9 basin groups based on the cyclogenesis location. This classification of basin is based on IBTrACS rule generally. But, for the case of the Southern Hemisphere, we determined by reference to the cluster analysis result which is reported by Ramsay et al. (2012). Then we calculated the centroid of frequency of TC occurrence at each TC development phase (cyclogenesis, developing and cyclolysis phase) at each basin. After that we calculated future shifts of the centroid at every GCM results. Finally we calculated ensemble average of them with considering the reproducibility weight of each GCM in present climate condition. Table 5 shows ensemble average centroid shift at each TC development phase at each basin. However we omitted 


\begin{tabular}{|c|c|c|}
\hline \multicolumn{3}{|c|}{ Table 4. Annual mean global cyclogenesis number $(\sharp /$ year $)$} \\
\hline Case & Mean & Standard Deviation \\
\hline SP0A & 84.4 & 17.0 \\
SPA & 84.7 & 8.8 \\
HP0A & 83.8 & 16.1 \\
HPA & 83.5 & 11.0 \\
HPA kf & 84.7 & 9.1 \\
HPA as & 84.8 & 11.0 \\
\hline SF0A & 65.4 & 9.3 \\
SFA & 71.8 & 7.1 \\
HF0A & 64.2 & 10.6 \\
HFA (a, b, c, d) & $(63.5,64.5,62.5,73.1)$ & $(9.2,10.4,7.8,8.3)$ \\
HFA kf $(a, b, c, d)$ & $(68.7,69.0,68.0,74.1)$ & $(9.1,7.8,9.0,7.4)$ \\
HFA as (a, b, c, d) & $(69.9,68.0,75.0,74.4)$ & $(10.8,10.1,10.9,11.9)$ \\
\hline
\end{tabular}

\begin{tabular}{|c|c|c|c|c|c|c|c|c|c|}
\hline \multicolumn{1}{|c|}{ Table 5. Each TC development phase centroid shift at each basin } \\
\hline \multicolumn{1}{|c|}{ Future shift of cyclogenesis location centroid } \\
\hline Basin & NWI & NEI & NWP & NEP & NA & SI & AR & SP & SA \\
\hline Longitudinal shift & -1.2 & 0.4 & 1.5 & -3.5 & 3.3 & -2.2 & -0.3 & 0.0 & - \\
Latitudinal shift & 1.4 & 0.6 & 0.4 & 0.6 & 0.2 & -1.5 & -0.3 & -0.1 & - \\
\hline \multicolumn{10}{|c|}{ Future shift of developing process location centroid } \\
\hline Basin & NWI & NEI & NWP & NEP & NA & SI & AR & SP & SA \\
\hline Longitudinal shift & -0.8 & 0.1 & 2.8 & -5.2 & 3.6 & -1.8 & 0.0 & 0.1 & - \\
Latitudinal shift & 1.5 & 1.0 & 1.2 & 1.4 & 1.0 & -1.7 & -0.5 & -0.1 & - \\
\hline \multicolumn{10}{|c|}{ Future shift of cyclolysis location centroid } \\
\hline Basin & NWI & NEI & NWP & NEP & NA & SI & AR & SP & SA \\
\hline Longitudinal shift & -0.5 & 0.5 & 3.0 & -3.6 & 4.5 & -0.6 & 0.3 & 0.0 & - \\
Latitudinal shift & 1.5 & 1.1 & 1.2 & 1.4 & 1.5 & -2.0 & -0.2 & 0.2 & - \\
\hline
\end{tabular}

$*$ Northward and eastward directions are positive, and unit is degree.

NWI:Northwest Indian Ocean, NEI:Northeast Indian Ocean

NWP:Northwest Pasific Ocean, NEP:Northeast Pasific Ocean

NA:North Atlantic Ocean, SI:South Indian Ocean, AR:Australian Region

SP:South Pasific Ocean, SA:South Atlantic Ocean

the result at the South Atlantic region because there is no historical TC data. Generally, the magnitude of longitudinal shift will be larger than that of latitudinal shift, and each centroid will shift to the center of basin. And future shift trend of each development phase is similar. We implemented these future shift trend into GSTCM by the horizontal movement of statistical relations at each basin.

Future change of TC intensityWe examined future change of TC translation speed and direction, but their change was smaller than deviation between different GCM results. On the other hand, TC central pressure shows significant future change trend. Briefly future TC central pressure will be small (TC will be strong) with increase of the latitude. Actually, the difference of future change between different GCM results is large at high latitude region, but all results show future TC will be strong with increase of the latitude. In this study, we estimated the following polynomial curve, which describe ensemble average future change of central pressure, from every GCM data with considering the weight of their reproducibility.

$$
\Delta p=-2.09 \times 10^{-6} x^{4}+5.95 \times 10^{-5} x^{3}+2.18 \times 10^{-4} x^{2}+5.12 \times 10^{-2} x-7.02 \times 10^{-1}
$$

Here $x$ is latitude. The reason why future TC becomes strong at high latitude region is possibly because sea surface temperature will be increase at high and middle latitude and the attenuation effect of TC will be decrease.

Finally, we implemented ensemble averaged future change trend described above into GSTCM which was calibrated by historical TC data. However, only future change of TC central pressure was added to results after Monte-Carlo simulation. 


\section{ASSESSMENT OF FUTURE TC AND THEIR STORM SURGE CHARACTERISTICS}

In this study, five thousand year simulations in present and future climate condition were conducted by GSTCM. Fig. 5 shows future change of annual mean TC arrival frequency. Globally, TC arrival frequency will decrease under the influence of the decrease of cyclogenesis number. However, future changes are not uniform at all regions. Furthermore, in some region, TC arrival frequency will be increase under the influence of the TC track shift. As seen above, TC arrival frequency is determined as a consequence of competition of future change of cyclogenesis number and track shift. For example, at southern part of the North-West Pacific Ocean (around the Philippine island and the south China), annual mean TC arrival frequency will decrease about 2 TCs per year. In this region, TC track shift effect is larger than that of other region relatively because arrival TC frequency is very high.

Next, Fig. 6 shows future change of mean TC central pressure. Generally, mean future TC central pressure will decrease (future TC will be strong) with the increase of latitude. However, the magnitude of future change of central pressure is smaller than $10 \mathrm{hPa}$ in most region except for SI and NA. According to the equation (5), average future change trend of central pressure is negative every latitude. Nevertheless, the central pressure of future TC will increase at some region. These local future changes are caused by the TC track shift effect at each basin. For example, there is wide TC developing zone around the Philippine island, and TC central pressure is low on average, but TC developing zone will move northeastward in the future. As a result, mean TC central pressure will increase there. Thus local future change of TC central pressure is also sensitive to the future change of TC track not only to synoptic latitudinal future change trend.

On the other hand, Fig. 7 shows future change of extreme TC central pressure. For estimation of this extreme value, we assumed annual minimum central pressure distribution satisfy Generalized Extreme Value PDF. The return period of this extreme value is 100 year. Generally, future change pattern of extreme TC central pressure is similar to that of average TC. However the extreme value is more sensitive to future change of TC tracks and cyclogenesis number. In addition, the magnitude of future change of extreme value is larger than that of mean TC in some regions. This is because the extreme value is sensitive to the future change of TC arrival frequency.

From these results, it is shown that the local future change is considerably sensitive to future TC track shift. In addition, the magnitude of future change of extreme TC is different to that of mean TC. Therefore, if we project future storm surge height at local place from mean future change trend of TC, its result would be different to what we want. This knowledge is supported the importance of risk assessment of extreme storm surge using GSTCM.

By using GSTCM simulation results, we can estimate the future change of storm surge height at local port. Actually storm surge height is determined from not only TC central pressure but also wind speed, arrival angle and TC translation speed. Therefore we analyzed in easy manner by using the following empirical equation.

$$
h=a(1010-P)+b W^{2} \cos \theta
$$

Here $P$ is central pressure, $W$ is wind speed and $\theta$ is arrival angle. Other coefficients $(a, b)$ were determined from historical record at each port. Wind speed distribution around TC can be estimated from idealized TC pressure model and gradient wind model. For example, we estimated future change of storm surge height of 100 year return period $h_{100}$ at major port in Japan as a trial. As a result, future storm surge height $h_{100}$ was $0.27 \mathrm{~m}$ higher than that of present climate at Tokyo port. And future $h_{100}$ was $0.38 \mathrm{~m}$ higher than that of present climate at Nagoya port. Other port results varied between +0.1 and $+0.4 \mathrm{~m}$. However these future projection values are sensitive to the amount of future TC track shift. Actually they varied considerably between each GCM. Therefore these future projection values still have the uncertainty. So we have to estimate the range of confidence from now on.

\section{CONCLUSION}

First, Global Stochastic Tropical Cyclone Model based on temporal correlation using cluster analysis was developed, and its performance was verified. Especially, bimodal joint PDF model performance is superior to that of unimodal joint PDF model for TC central pressure and translation direction.

Second, future change trends of TC were estimated from 15 ensemble GCM results. As a result, the following trends were significant. 


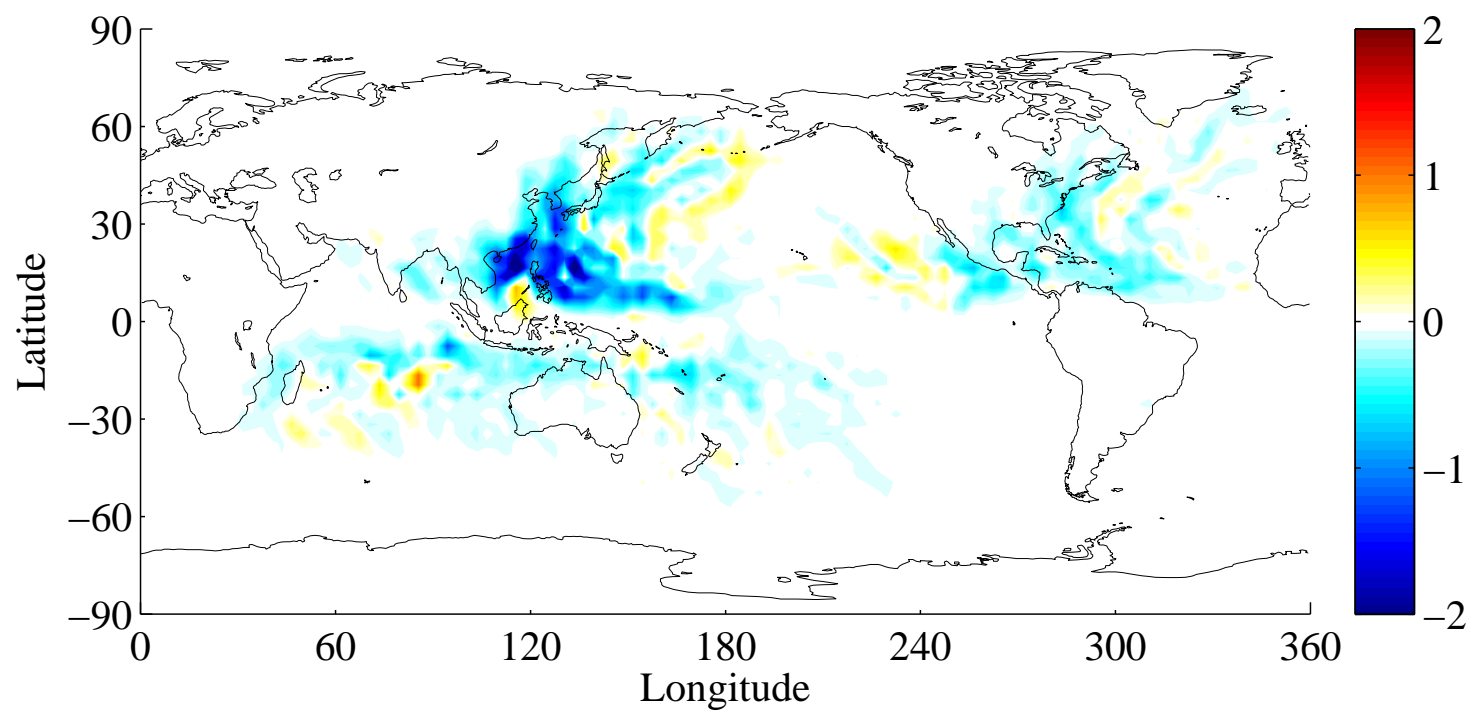

Figure 5: Future change of annual mean TC arrival frequency ( $\sharp /$ year)

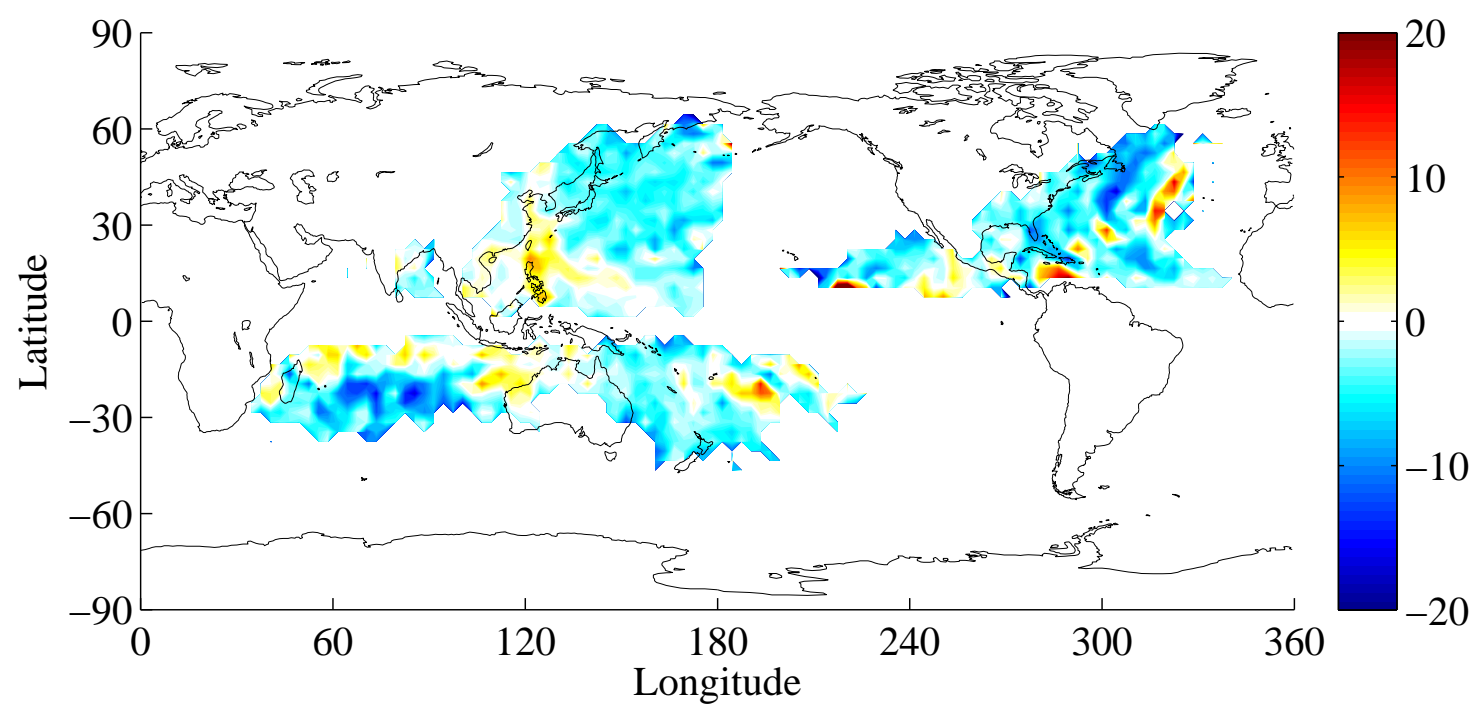

Figure 6: Future change of mean TC central pressure (hPa) 


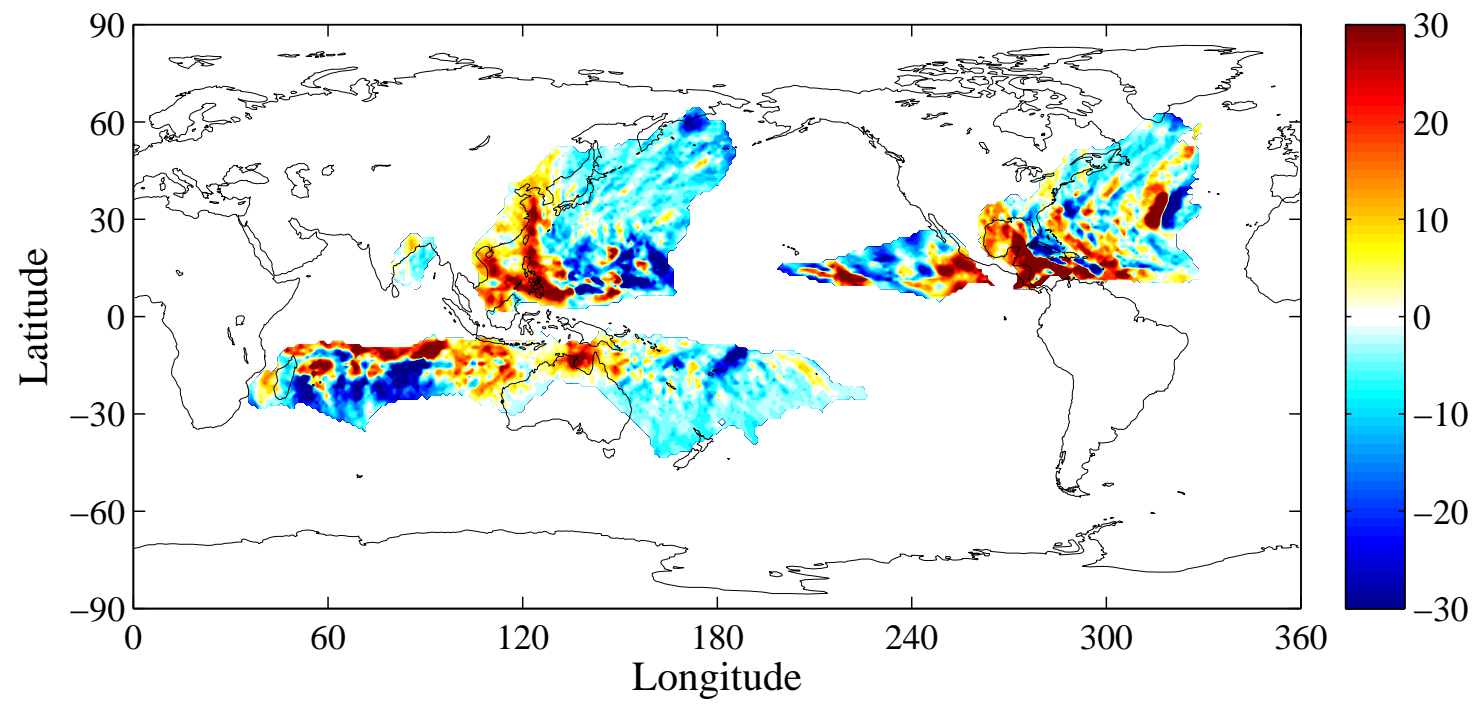

Figure 7: Future change of extreme TC central pressure of 100 year return period (hPa)

1. Global annual mean cyclogenesis number will decrease $-17 \%$ on ensemble average.

2. Generally, the magnitude of longitudinal shift will be larger than that of latitudinal shift, and each centroid will shift to the center of basin.

3. Future TC central pressure will decrease with the increase of latitude.

Finally, as a result of GSTCM simulation, it is clear that TC frequency will decrease globally but will increase in some region due to the track shift effect. Forced synoptic latitudinal future change trend of central pressure stands out globally in GSTCM result, but track shift effect is also not negligible locally. Especially, future change pattern of extreme TC central pressure is similar to that of mean TC, but extreme value is more sensitive to future change of TC tracks and cyclogenesis number. Therefore, we have to use the GSTCM result to assess the risk of extreme storm surge. Future change of extreme storm surge heights of 100 year return period at Japanese major port were estimated and their orders varied between +0.1 and $+0.4 \mathrm{~m}$.

\section{ACKNOWLEDGEMENTS}

This research was supported by the KAKUSHIN program of the Ministry of Education, Culture, Sports, Science, and Technology of Japan (MEXT).

\section{References}

T. M. Hall and S. Jewson. Statistical modeling of north atlantic tropical cyclone tracks. Tellus, 59A: 486-498, 2007.

M. K. James and L. B. Mason. Synthetic tropical cyclone database. Journal of Waterway, Port, Coastal, and Ocean Engineering, 131(4):181-192, 2005.

T. R. Knutson, J. L. McBride, J. Chan, K. Emanuel, G. Holland, C. Landsea, I. Held, J. P. Kossin, A. K. Srivastava, and M. Sugi. Tropical cyclones and climate change. Nature Geoscience, 3(779):157-163, 2010.

H. Murakami, B. Wang, and A. Kitoh. Future change of western north pacific typhoons projections by a 20-km-mesh global atmospheric model. Journal of Climate, 24:1154-1169, 2011.

S. Nakajo, N. Mori, T. Yasuda, and H. Mase. Prediction of future tropical cyclone characteristics using global stochastic tropical cyclone model. Proceedings of Asia Pacific Coasts 2011, (41):9pp., 2011. 
H. A. Ramsay, S. J. Camargo, and D. Kim. Cluster analysis of tropical cyclone tracks in the southern hemisphere. Climate dynamics, 39:897-917, 2012.

P. J. Vickery, P. F. Skerlj, and L. A. Twisdale. Simulation of hurricane risk in the u.s. using empirical track model. Journal of Structural Engineering, 126(10):1222-1237, 2000. 